\title{
A Novel Synthesis of 5-Functionalized Oxazolidin-2-ones from Enantiomerically Pure 2-Substituted $N-[(R)-(+)-\alpha$-Methylbenzyl $]$ aziridines
}

Tae Bo Sim, Se Hun Kang, Kun Su Lee, and Won Koo Lee*

Department of Chemistry, Sogang University, Seoul 121-742, Korea

Hoseop Yun, Yongkwan Dong

Department of Molecular Science and Technology, Ajou University, Suwon, 442-749, Korea

\section{Hyun-Joon $\mathrm{Ha}^{*}$}

Department of Chemistry, Hankuk University of Foreign Studies, Yongin, 449-791, Korea

\section{Supporting Information}

X-Ray crystallographic data for $\mathbf{6 a}$ and $\mathbf{6 b}$.

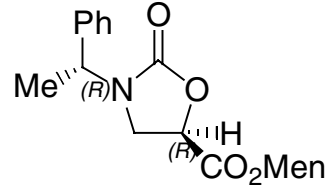

$6 a$

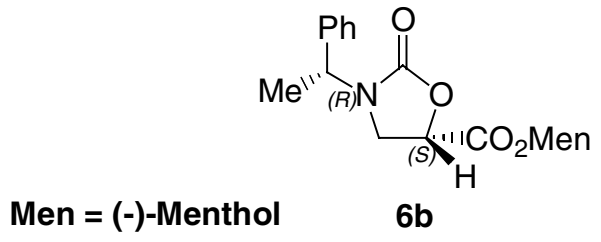

$6 b$

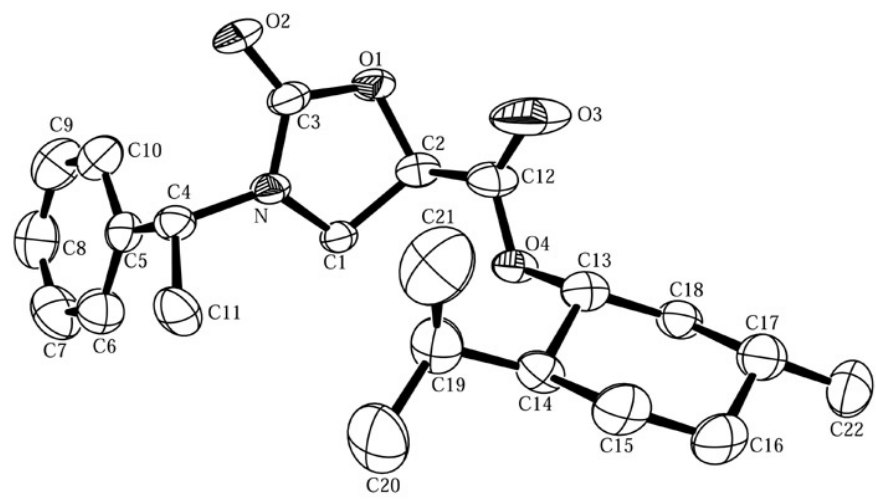

6a 


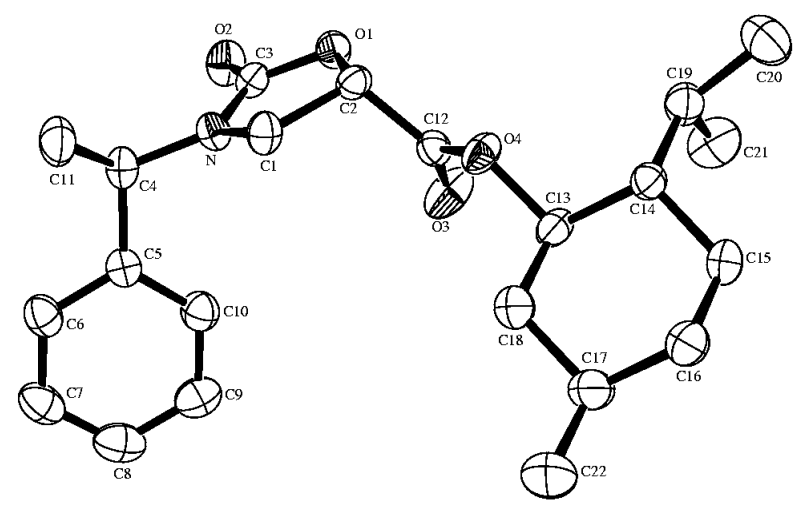

6b

\section{Crystallographic studies of $6 a$ and $6 b$.}

Preliminary examination and data collection for compounds $\mathbf{6 a}$ and $\mathbf{6 b}$ were performed with MoK radiation ( $=0.7107 \AA$ ) on an MXC3 diffractometer (MAC Science) at $130(1) \mathrm{K}$. Cell constants and an orientation matrix for data collection were determined from the leastsquares analysis, using setting angles of 26 reflections in the range of $20 \quad 2 \quad 28$ for $\mathbf{6 a}$ and 22 reflections in the range of $20 \quad 2 \quad 28$ for $\mathbf{6 b}$. For both compounds, intensity data were collected with the $\quad-2$ scan with $3 \quad 2 \quad 50$ and two standard reflections monitored every 100 reflections throughout the data collection process showed no significant trends.

The 6a crystallizes in the space group $\mathrm{D}_{2}{ }^{4}-P 2{ }_{1}{ }_{2} 2_{1}$ of the orthorhombic system with four formula units in a cell dimensions: $a=15.237(4) \AA, \quad b=22.307(5) \AA, \quad c=6.417(2) \AA$, and $\mathrm{V}=2186.2(11) \AA^{3}$. Equivalent reflection has been checked for Laue symmetry. The transparent needle shaped crystal of dimension $0.64 \times 0.18 \times 0.15 \mathrm{~mm}^{3}$ was used to collect 1800 unique $\left(\mathrm{F}_{\mathrm{o}}^{2}\right.$ 0) and $1041\left(\mathrm{~F}_{\mathrm{o}}^{2} 2\left(\mathrm{~F}_{\mathrm{o}}^{2}\right)\right)$ reflections. The $\mathbf{6 b}$ crystallizes in the space group $\mathrm{C}_{2}{ }^{2}-P 2_{1}$ of the monoclinic system with two formula units in a cell dimensions: $\mathrm{a}=14.167(5) \AA, \quad b=6.346(2) \AA, \quad c=12.483(4) \AA, \quad=108.64(2), \quad$ and $\quad V=1063.4(6) \AA^{3}$. The transparent polyhedron crystal of dimension $0.84 \times 0.28 \times 0.26 \mathrm{~mm}^{3}$ was used to collect 1667 
unique $\left(\mathrm{F}_{\mathrm{o}}^{2} 0\right)$ and $1308\left(\mathrm{~F}_{\mathrm{o}}^{2} 2\left(\mathrm{~F}_{\mathrm{o}}^{2}\right)\right)$ reflections.

The initial positions for all non-hydrogen atoms were obtained by using direct methods of the SHELXS-86 program (1). The structure was refined with the use of the SHELXL-97 program (2). Positional and thermal parameters for non-hydrogen atoms were refined using a full-matrix least-squares refinement procedure. Atomic positions of hydrogen atoms were generated with riding model technique of SHELXL-97 (2). The final cycle of refinement performed with wR2 $=0.1301$ on $\mathrm{F}_{\mathrm{o}}^{2}\left(\mathrm{~F}_{\mathrm{o}}^{2}>0\right)$ for $\mathbf{6 a}$ and $w \mathrm{R} 2=0.0838$ on $\mathrm{F}_{\mathrm{o}}^{2}\left(\mathrm{~F}_{\mathrm{o}}^{2}>0\right)$ for $\mathbf{6 b}$ and the conventional $\mathrm{R}$ index based on the reflections having $\mathrm{F}_{\mathrm{o}}^{2}>2\left(\mathrm{~F}_{\mathrm{o}}^{2}\right)$ is 0.0584 for $\mathbf{6 a}$ and 0.0393 for 6b. Crystal data and crystallographic details are described in Table 1. None of the structures shows additional potential symmetry, as tested by the MISSYM algorithm in the PLATON suite of program $(3,4)$. Final values of atomic parameters and equivalent isotropic displacement parameters are given in Table 2 for $\mathbf{6 a}$ and Table 3 for $\mathbf{6 b}$. Final anisotropic displacement parameters appear Table 4 for non-hydrogen atoms. Bond distances and angles are given in Table 5 and 6, respectively.

(1) Sheldrick, G. M. Acta Crystallogr. 1990, A46, 467 - 473.

(2) Sheldrick, G. M. SHELXL97. Program for the Refinement of Crystal Structure.

University of Göttingen, Germany, 1997.

(3) Le Page, Y. J. Appl. Crystallogr., 1987, 20, 264-269.

(4) Spek, A. L. Acta Crystallogr., A46, 1990, C-34. 
Table 1. Crystal Data and Structure Refinement for $\mathbf{6 a}$ and $\mathbf{6 b}$.

\begin{tabular}{|c|c|c|}
\hline & $\mathbf{6 a}$ & $6 \mathbf{b}$ \\
\hline Formula & $\mathrm{C}_{22} \mathrm{H}_{31} \mathrm{NO}_{4}$ & $\mathrm{C}_{22} \mathrm{H}_{31} \mathrm{NO}_{4}$ \\
\hline Formula Weight, amu & 373.48 & 373.48 \\
\hline Space Group & $\mathrm{D}_{2}^{4}-P 2{ }_{1} 2_{1} 2_{1}$ & $\mathrm{C}_{2}^{2}-P 2_{1}$ \\
\hline $\mathrm{a}, \AA$ & $15.273(4)$ & $14.167(5)$ \\
\hline $\mathrm{b}, \AA$ & $22.307(5)$ & $6.346(2)$ \\
\hline $\mathrm{c}, \AA$ & $6.417(2)$ & $12.483(4)$ \\
\hline , deg. & & $108.64(2)$ \\
\hline $\mathrm{V}, \AA^{3}$ & $2186.2(11)$ & $1063.4(6)$ \\
\hline Radiation & \multicolumn{2}{|c|}{ Graphite monochromated MoK $\quad(=0.7107 \AA)$} \\
\hline Z, Temperature, $\mathrm{K}$ & $4,130(1)$ & $2,130(1)$ \\
\hline Scan Type & -2 & -2 \\
\hline Scan Rate & $4.0 / \mathrm{min}$ (in ) & $5.0 / \mathrm{min}$ (in ) \\
\hline Scan Width, deg. & $1.0+0.35 \tan$ & $0.9+0.35 \tan$ \\
\hline 2 max, deg. & 50.0 & 50.0 \\
\hline \multirow[t]{2}{*}{ No. of Reflections Measured } & 1806 & 1742 \\
\hline & 1800 & $1667\left(\mathrm{R}_{i n t}=0.0145\right)$ \\
\hline No. Observations $\left(\mathrm{F}_{\mathrm{o}}^{2} 0\right)$ & 1800 & 1667 \\
\hline No. Unique data with $\mathrm{F}_{\mathrm{o}}^{2}>2 \quad\left(\mathrm{~F}_{\mathrm{o}}^{2}\right)$ & 1041 & 1308 \\
\hline No. Variables & 249 & 249 \\
\hline Reflection / Parameter Ratio & 13.83 & 14.94 \\
\hline $\mathrm{wR} 2\left(\mathrm{~F}_{\mathrm{o}}^{2} 0\right)$ & 0.1301 & 0.0838 \\
\hline $\mathrm{R}\left(\right.$ on $\mathrm{F}_{\mathrm{o}}^{2}$ for $\left.\mathrm{F}_{\mathrm{o}}^{2}>2 \quad\left(\mathrm{~F}_{\mathrm{o}}^{2}\right)\right)$ & 0.0584 & 0.0393 \\
\hline Goodness of Fit Indicator & 1.029 & 1.026 \\
\hline Max Shift / Error in Final Cycle & $<0.0005$ & $<0.0005$ \\
\hline Maximum peak in Final Diff. Map & $0.217 \mathrm{e}^{-} / \AA^{3}$ & $0.112 \mathrm{e}^{-} / \AA^{3}$ \\
\hline Minimum peak in Final Diff. Map & $-0.188 \mathrm{e}^{-} / \AA^{3}$ & $0.097 \mathrm{e}^{-/} \AA^{3}$ \\
\hline
\end{tabular}


Table 2. Atomic Coordinates (x 10 ${ }^{4}$ ) and Equivalent Isotropic Displacement Parameters $\left(\AA^{2} \times 10^{3}\right)$ for $\mathbf{6 a}$.

\begin{tabular}{|c|c|c|c|c|}
\hline Atom & $\mathrm{x}$ & $\mathrm{y}$ & $\mathrm{z}$ & $\mathrm{U}_{\mathrm{eq}}{ }^{a}$ \\
\hline $\mathrm{C}(1)$ & $1703(4)$ & $4029(2)$ & $12171(8)$ & $47(2)$ \\
\hline$C(2)$ & $1928(4)$ & $4625(3)$ & $11146(9)$ & $58(2)$ \\
\hline$C(3)$ & $1348(4)$ & $4019(3)$ & $8679(10)$ & $63(2)$ \\
\hline $\mathrm{C}(4)$ & $777(4)$ & $3172(3)$ & 10693(9) & $57(2)$ \\
\hline$C(5)$ & $1470(4)$ & $2692(3)$ & $10808(11)$ & $63(2)$ \\
\hline$C(6)$ & $1621(5)$ & $2349(3)$ & $12557(13)$ & $87(2)$ \\
\hline$C(7)$ & $2266(7)$ & $1906(4)$ & $12559(18)$ & $114(3)$ \\
\hline$C(8)$ & $2762(6)$ & $1810(4)$ & $10830(2)$ & $105(3)$ \\
\hline$C(9)$ & $2625(6)$ & $2139(4)$ & $9106(17)$ & $104(3)$ \\
\hline$C(10)$ & $1988(5)$ & $2575(3)$ & $9079(12)$ & $82(2)$ \\
\hline $\mathrm{C}(11)$ & $108(4)$ & $3156(3)$ & $12439(13)$ & $81(2)$ \\
\hline$C(12)$ & $1324(5)$ & $5139(3)$ & $11582(10)$ & $68(2)$ \\
\hline $\mathrm{C}(13)$ & $390(4)$ & $5600(3)$ & $14166(10)$ & $58(2)$ \\
\hline$C(14)$ & $-322(4)$ & $5275(3)$ & $15347(10)$ & $61(2)$ \\
\hline$C(15)$ & $-971(4)$ & $5739(3)$ & $16163(11)$ & $81(2)$ \\
\hline$C(16)$ & $-534(5)$ & $6211(3)$ & $17482(12)$ & $86(2)$ \\
\hline $\mathrm{C}(17)$ & $207(5)$ & $6537(3)$ & $16357(12)$ & $80(2)$ \\
\hline$C(18)$ & $844(4)$ & $6078(3)$ & $15466(9)$ & $64(2)$ \\
\hline$C(19)$ & $-727(5)$ & $4769(3)$ & $14100(13)$ & $86(2)$ \\
\hline $\mathrm{C}(20)$ & $-1258(5)$ & $4356(3)$ & $15500(14)$ & 139(4) \\
\hline $\mathrm{C}(21)$ & $-1286(6)$ & $4967(4)$ & $12237(16)$ & $152(4)$ \\
\hline $\mathrm{C}(22)$ & $661(5)$ & $6983(3)$ & $17724(14)$ & $112(3)$ \\
\hline $\mathrm{N}$ & $1165(3)$ & $3767(2)$ & $10541(7)$ & $52(1)$ \\
\hline $\mathrm{O}(1)$ & $1866(3)$ & $4502(2)$ & $8941(6)$ & $69(1)$ \\
\hline $\mathrm{O}(2)$ & 1101(4) & $3850(2)$ & $6984(6)$ & $98(2)$ \\
\hline $\mathrm{O}(3)$ & $1132(5)$ & $5498(3)$ & 10341(8) & $165(3)$ \\
\hline $\mathrm{O}(4)$ & $1052(2)$ & $5158(2)$ & $13538(6)$ & $56(1)$ \\
\hline $\mathrm{H}(1 \mathrm{~A})$ & 2222 & 3791 & 12444 & 57 \\
\hline $\mathrm{H}(1 \mathrm{~B})$ & 1377 & 4084 & 13455 & 57 \\
\hline $\mathrm{H}(2)$ & 2530 & 4738 & 11499 & 69 \\
\hline $\mathrm{H}(4)$ & 454 & 3105 & 9393 & 68 \\
\hline $\mathrm{H}(6)$ & 1289 & 2413 & 13752 & 104 \\
\hline
\end{tabular}


Table 2. Atomic Coordinates (x $10^{4}$ ) and Equivalent Isotropic Displacement Parameters $\left(\AA^{2} \times 10^{3}\right)$ for $\mathbf{6 a}$. (continued)

\begin{tabular}{|c|c|c|c|c|}
\hline Atom & $\mathrm{x}$ & $\mathrm{y}$ & $\mathrm{z}$ & $\mathrm{U}_{\mathrm{eq}}{ }^{a}$ \\
\hline $\mathrm{H}(7)$ & 2357 & 1675 & 13747 & 136 \\
\hline $\mathrm{H}(8)$ & 3196 & 1518 & 10846 & 126 \\
\hline $\mathrm{H}(9)$ & 2963 & 2072 & 7924 & 125 \\
\hline $\mathrm{H}(10)$ & 1902 & 2798 & 7870 & 98 \\
\hline $\mathrm{H}(11 \mathrm{~A})$ & -335 & 3453 & 12186 & 122 \\
\hline $\mathrm{H}(11 \mathrm{~B})$ & 393 & 3239 & 13741 & 122 \\
\hline $\mathrm{H}(11 \mathrm{C})$ & -157 & 2766 & 12496 & 122 \\
\hline $\mathrm{H}(13)$ & 138 & 5785 & 12919 & 69 \\
\hline $\mathrm{H}(14)$ & -44 & 5092 & 16568 & 74 \\
\hline $\mathrm{H}(15 \mathrm{~A})$ & -1259 & 5930 & 14993 & 98 \\
\hline $\mathrm{H}(15 \mathrm{~B})$ & -1416 & 5537 & 16985 & 98 \\
\hline $\mathrm{H}(16 \mathrm{~A})$ & -302 & 6023 & 18729 & 103 \\
\hline $\mathrm{H}(16 \mathrm{~B})$ & -970 & 6501 & 17914 & 103 \\
\hline $\mathrm{H}(17)$ & -50 & 6757 & 15184 & 96 \\
\hline $\mathrm{H}(18 \mathrm{~A})$ & 1272 & 6284 & 14606 & 76 \\
\hline $\mathrm{H}(18 \mathrm{~B})$ & 1155 & 5887 & 16604 & 76 \\
\hline H(19) & -243 & 4531 & 13538 & 104 \\
\hline $\mathrm{H}(20 \mathrm{~A})$ & -1766 & 4565 & 16000 & 209 \\
\hline $\mathrm{H}(20 \mathrm{~B})$ & -905 & 4233 & 16662 & 209 \\
\hline $\mathrm{H}(20 \mathrm{C})$ & -1437 & 4009 & 14723 & 209 \\
\hline $\mathrm{H}(21 \mathrm{~A})$ & -1788 & 5184 & 12728 & 229 \\
\hline $\mathrm{H}(21 \mathrm{~B})$ & -1475 & 4620 & 11475 & 229 \\
\hline $\mathrm{H}(21 \mathrm{C})$ & -945 & 5220 & 11341 & 229 \\
\hline $\mathrm{H}(22 \mathrm{~A})$ & 1126 & 7172 & 16957 & 169 \\
\hline $\mathrm{H}(22 \mathrm{~B})$ & 900 & 6782 & 18919 & 169 \\
\hline $\mathrm{H}(22 \mathrm{C})$ & 250 & 7282 & 18172 & 169 \\
\hline
\end{tabular}

${ }^{a} \mathrm{U}_{\mathrm{eq}}$ is defined as one third of the trace of the orthogonalized $\mathrm{U}_{\mathrm{ij}}$ tensor. 
Table 3. Atomic Coordinates (x 10 ${ }^{4}$ ) and Equivalent Isotropic Displacement Parameters $\left(\AA^{2} \times 10^{3}\right)$ for $\mathbf{6 b}$.

\begin{tabular}{|c|c|c|c|c|}
\hline Atom & $\mathrm{x}$ & $\mathrm{y}$ & $\mathrm{z}$ & $\mathrm{U}_{\mathrm{eq}}{ }^{a}$ \\
\hline $\mathrm{C}(1)$ & $11400(2)$ & $3542(6)$ & $9426(3)$ & $51(1)$ \\
\hline$C(2)$ & $11279(2)$ & $5182(6)$ & $10278(3)$ & $45(1)$ \\
\hline$C(3)$ & $9937(3)$ & $5454(6)$ & $8658(3)$ & $47(1)$ \\
\hline $\mathrm{C}(4)$ & $10313(3)$ & $2882(6)$ & $7375(3)$ & $52(1)$ \\
\hline$C(5)$ & 10904(2) & $3629(6)$ & $6622(3)$ & $47(1)$ \\
\hline$C(6)$ & 10892(3) & $2453(7)$ & $5681(3)$ & $62(1)$ \\
\hline$C(7)$ & 11371(3) & $3140(9)$ & 4939(3) & $75(1)$ \\
\hline$C(8)$ & $11875(3)$ & $5011(10)$ & $5135(4)$ & $81(2)$ \\
\hline$C(9)$ & 11902(3) & $6201(9)$ & $6065(4)$ & $79(1)$ \\
\hline$C(10)$ & 11421(3) & $5489(7)$ & $6799(3)$ & $61(1)$ \\
\hline $\mathrm{C}(11)$ & $10412(3)$ & $526(7)$ & $7616(3)$ & $73(1)$ \\
\hline$C(12)$ & $12088(3)$ & $6814(6)$ & $10516(3)$ & $46(1)$ \\
\hline$C(13)$ & $13801(2)$ & $7333(6)$ & $11620(3)$ & $50(1)$ \\
\hline $\mathrm{C}(14)$ & $14182(2)$ & $7751(6)$ & $12883(3)$ & $46(1)$ \\
\hline$C(15)$ & $15239(3)$ & $8617(7)$ & 13194(3) & $63(1)$ \\
\hline$C(16)$ & $15930(3)$ & $7219(8)$ & $12803(3)$ & $68(1)$ \\
\hline $\mathrm{C}(17)$ & $15552(3)$ & $6888(8)$ & $11538(3)$ & $70(1)$ \\
\hline$C(18)$ & $14497(2)$ & $5981(8)$ & $11210(3)$ & $67(1)$ \\
\hline$C(19)$ & $13468(3)$ & $9100(6)$ & $13289(3)$ & $59(1)$ \\
\hline$C(20)$ & $13740(3)$ & $9071(9)$ & 14571(3) & $91(2)$ \\
\hline$C(21)$ & $13360(3)$ & $11370(8)$ & 12861(4) & $90(2)$ \\
\hline $\mathrm{C}(22)$ & $16233(3)$ & $5498(12)$ & $11133(4)$ & $121(2)$ \\
\hline $\mathrm{N}$ & $10565(2)$ & $4046(4)$ & $8439(2)$ & $45(1)$ \\
\hline $\mathrm{O}(1)$ & $10331(2)$ & $6161(4)$ & $9746(2)$ & $53(1)$ \\
\hline $\mathrm{O}(2)$ & $9151(2)$ & $6080(5)$ & $8038(2)$ & $66(1)$ \\
\hline $\mathrm{O}(3)$ & $12065(2)$ & $8360(5)$ & $9968(2)$ & $82(1)$ \\
\hline $\mathrm{O}(4)$ & $12863(2)$ & $6179(4)$ & $11367(2)$ & $55(1)$ \\
\hline $\mathrm{H}(1 \mathrm{~A})$ & 12028 & 3712 & 9282 & 61 \\
\hline $\mathrm{H}(1 \mathrm{~B})$ & 11352 & 2117 & 9685 & 61 \\
\hline $\mathrm{H}(2)$ & 11283 & 4489 & 10982 & 54 \\
\hline $\mathrm{H}(4)$ & 9609 & 3160 & 6968 & 62 \\
\hline $\mathrm{H}(6)$ & 10554 & 1174 & 5547 & 75 \\
\hline
\end{tabular}


Table 3. Atomic Coordinates (x 10 ${ }^{4}$ ) and Equivalent Isotropic Displacement Parameters $\left(\AA^{2} \times 10^{3}\right)$ for $\mathbf{6 b}$. (continued)

\begin{tabular}{|c|c|c|c|c|}
\hline Atom & $\mathrm{x}$ & $\mathrm{y}$ & $\mathrm{z}$ & $\mathrm{U}_{\mathrm{eq}}{ }^{a}$ \\
\hline $\mathrm{H}(7)$ & 11351 & 2334 & 4309 & 90 \\
\hline $\mathrm{H}(8)$ & 12200 & 5480 & 4640 & 97 \\
\hline $\mathrm{H}(9)$ & 12243 & 7477 & 6198 & 94 \\
\hline $\mathrm{H}(10)$ & 11448 & 6295 & 7430 & 73 \\
\hline $\mathrm{H}(11 \mathrm{~A})$ & 10043 & 156 & 8114 & 109 \\
\hline $\mathrm{H}(11 \mathrm{~B})$ & 11101 & 176 & 7966 & 109 \\
\hline $\mathrm{H}(11 \mathrm{C})$ & 10153 & -238 & 6919 & 109 \\
\hline $\mathrm{H}(13)$ & 13685 & 8676 & 11211 & 60 \\
\hline $\mathrm{H}(14)$ & 14228 & 6380 & 13258 & 55 \\
\hline $\mathrm{H}(15 \mathrm{~A})$ & 15219 & 10003 & 12859 & 76 \\
\hline $\mathrm{H}(15 \mathrm{~B})$ & 15505 & 8778 & 14008 & 76 \\
\hline $\mathrm{H}(16 \mathrm{~A})$ & 16586 & 7856 & 13013 & 82 \\
\hline $\mathrm{H}(16 \mathrm{~B})$ & 15991 & 5865 & 13179 & 82 \\
\hline $\mathrm{H}(17)$ & 15516 & 8266 & 11173 & 84 \\
\hline $\mathrm{H}(18 \mathrm{~A})$ & 14233 & 5856 & 10394 & 80 \\
\hline $\mathrm{H}(18 \mathrm{~B})$ & 14525 & 4579 & 11528 & 80 \\
\hline H(19) & 12808 & 8449 & 12988 & 71 \\
\hline $\mathrm{H}(20 \mathrm{~A})$ & 14370 & 9761 & 14900 & 137 \\
\hline $\mathrm{H}(20 \mathrm{~B})$ & 13787 & 7638 & 14830 & 137 \\
\hline $\mathrm{H}(20 \mathrm{C})$ & 13236 & 9794 & 14791 & 137 \\
\hline $\mathrm{H}(21 \mathrm{~A})$ & 13194 & 11374 & 12053 & 135 \\
\hline $\mathrm{H}(21 \mathrm{~B})$ & 13977 & 12106 & 13191 & 135 \\
\hline $\mathrm{H}(21 \mathrm{C})$ & 12842 & 12058 & 13071 & 135 \\
\hline $\mathrm{H}(22 \mathrm{~A})$ & 16202 & 4081 & 11389 & 181 \\
\hline $\mathrm{H}(22 \mathrm{~B})$ & 16904 & 6009 & 11430 & 181 \\
\hline $\mathrm{H}(22 \mathrm{C})$ & 16026 & 5519 & 10322 & 181 \\
\hline
\end{tabular}

${ }^{a} \mathrm{U}_{\mathrm{eq}}$ is defined as one third of the trace of the orthogonalized $\mathrm{U}_{\mathrm{ij}}$ tensor. 
Table 4. Anisotropic displacement parameters $\left(\AA^{2} \times 10^{3}\right)$ for $\mathbf{6 a}$ and $\mathbf{6 b}$.

\begin{tabular}{|c|c|c|c|c|c|c|}
\hline Atom & $\mathrm{U}_{11}$ & $\mathrm{U}_{22}$ & $\mathrm{U}_{33}$ & $\mathrm{U}_{23}$ & $\mathrm{U}_{13}$ & $\mathrm{U}_{12}$ \\
\hline \multicolumn{7}{|c|}{$6 a$} \\
\hline $\mathrm{C}(1)$ & $55(4)$ & $49(4)$ & $37(3)$ & $0(3)$ & $1(3)$ & $7(3)$ \\
\hline$C(2)$ & $53(4)$ & $73(4)$ & $47(4)$ & $2(4)$ & $4(3)$ & $4(4)$ \\
\hline$C(3)$ & $75(5)$ & $75(5)$ & $37(4)$ & $-4(4)$ & $0(4)$ & $-13(4)$ \\
\hline $\mathrm{C}(4)$ & $55(4)$ & $69(4)$ & $47(4)$ & $0(4)$ & $5(4)$ & $-15(4)$ \\
\hline$C(5)$ & $67(4)$ & $61(4)$ & $60(5)$ & $-8(4)$ & $-3(5)$ & $-14(4)$ \\
\hline$C(6)$ & $94(6)$ & $84(6)$ & $82(6)$ & $6(5)$ & $-8(5)$ & $-2(5)$ \\
\hline$C(7)$ & $119(8)$ & $90(7)$ & 131(9) & $31(7)$ & $-33(8)$ & $-3(7)$ \\
\hline $\mathrm{C}(8)$ & $88(6)$ & $87(7)$ & $139(9)$ & $-22(8)$ & $-12(8)$ & $6(5)$ \\
\hline $\mathrm{C}(9)$ & $92(7)$ & $116(8)$ & $103(7)$ & $-28(7)$ & $-4(6)$ & $14(6)$ \\
\hline$C(10)$ & $79(5)$ & $94(6)$ & $72(5)$ & $-17(5)$ & $1(5)$ & $-4(5)$ \\
\hline$C(11)$ & $69(4)$ & $75(5)$ & $99(5)$ & $10(5)$ & $15(4)$ & $-18(4)$ \\
\hline$C(12)$ & $73(5)$ & $85(5)$ & $47(4)$ & $17(4)$ & $-7(4)$ & $14(4)$ \\
\hline$C(13)$ & $49(4)$ & $69(4)$ & $55(4)$ & $9(4)$ & $-1(4)$ & $18(4)$ \\
\hline$C(14)$ & $49(4)$ & $64(4)$ & $71(4)$ & $11(4)$ & $15(4)$ & $8(4)$ \\
\hline$C(15)$ & $54(4)$ & $99(5)$ & $91(5)$ & $-2(5)$ & $22(4)$ & $18(4)$ \\
\hline$C(16)$ & $82(5)$ & $93(5)$ & $83(5)$ & $-2(5)$ & $16(5)$ & $41(5)$ \\
\hline$C(17)$ & $88(5)$ & $73(5)$ & $78(5)$ & $7(5)$ & $15(5)$ & $25(5)$ \\
\hline$C(18)$ & $67(4)$ & $65(4)$ & $59(4)$ & $8(4)$ & $11(4)$ & $2(4)$ \\
\hline$C(19)$ & $61(4)$ & $86(5)$ & $112(6)$ & $-11(6)$ & $19(5)$ & $-12(4)$ \\
\hline$C(20)$ & 111(7) & $122(7)$ & $186(10)$ & $-20(7)$ & $59(8)$ & $-45(6)$ \\
\hline$C(21)$ & $115(7)$ & 187(9) & $156(9)$ & $-31(8)$ & $-50(8)$ & $-28(8)$ \\
\hline$C(22)$ & $142(7)$ & $74(5)$ & $121(7)$ & $-22(6)$ & $36(7)$ & $1(6)$ \\
\hline $\mathrm{N}$ & $62(3)$ & $62(3)$ & $34(3)$ & $3(3)$ & $-6(3)$ & $-13(3)$ \\
\hline $\mathrm{O}(1)$ & $86(3)$ & $78(3)$ & $43(3)$ & $-1(3)$ & $24(3)$ & $-23(3)$ \\
\hline $\mathrm{O}(2)$ & $160(5)$ & $102(4)$ & $32(2)$ & $-8(3)$ & $-11(3)$ & $-28(4)$ \\
\hline $\mathrm{O}(3)$ & $252(8)$ & $177(6)$ & $65(4)$ & $48(4)$ & $48(5)$ & $142(6)$ \\
\hline $\mathrm{O}(4)$ & $58(3)$ & $65(3)$ & $44(2)$ & $11(2)$ & $7(2)$ & $11(2)$ \\
\hline
\end{tabular}


Table 4. Anisotropic displacement parameters $\left(\AA^{2} \times 10^{3}\right)$ for OX (I) and $\mathbf{6 b}$. (continued)

\begin{tabular}{|c|c|c|c|c|c|c|}
\hline Atom & $\mathrm{U}_{11}$ & $\mathrm{U}_{22}$ & $\mathrm{U}_{33}$ & $\mathrm{U}_{23}$ & $\mathrm{U}_{13}$ & $\mathrm{U}_{12}$ \\
\hline \multicolumn{7}{|c|}{$6 b$} \\
\hline $\mathrm{C}(1)$ & $45(2)$ & $47(2)$ & $53(2)$ & $-5(2)$ & $4(2)$ & $4(2)$ \\
\hline$C(2)$ & $36(2)$ & $50(2)$ & $48(2)$ & $0(2)$ & $12(2)$ & $-4(2)$ \\
\hline$C(3)$ & $42(2)$ & $44(2)$ & $54(2)$ & $-2(2)$ & $13(2)$ & $-4(2)$ \\
\hline $\mathrm{C}(4)$ & $51(2)$ & $47(2)$ & $49(2)$ & $-7(2)$ & $5(2)$ & $1(2)$ \\
\hline$C(5)$ & $49(2)$ & $42(2)$ & $44(2)$ & $4(2)$ & $8(2)$ & $8(2)$ \\
\hline$C(6)$ & $59(2)$ & $69(3)$ & $53(2)$ & $-8(2)$ & $12(2)$ & $6(2)$ \\
\hline$C(7)$ & $71(3)$ & 101(4) & $52(3)$ & $-3(3)$ & $20(2)$ & $13(3)$ \\
\hline $\mathrm{C}(8)$ & $67(3)$ & $111(5)$ & $66(3)$ & $18(3)$ & $26(2)$ & $14(3)$ \\
\hline $\mathrm{C}(9)$ & $78(3)$ & $77(3)$ & $85(3)$ & $10(3)$ & $32(3)$ & $-10(3)$ \\
\hline$C(10)$ & $70(3)$ & $52(3)$ & $64(2)$ & $-2(2)$ & $25(2)$ & $1(2)$ \\
\hline $\mathrm{C}(11)$ & $103(3)$ & $51(3)$ & $60(2)$ & $-9(2)$ & $22(2)$ & $-16(3)$ \\
\hline$C(12)$ & $48(2)$ & $50(3)$ & $37(2)$ & $0(2)$ & $11(2)$ & $0(2)$ \\
\hline$C(13)$ & $42(2)$ & $55(3)$ & $48(2)$ & $7(2)$ & $7(2)$ & $-13(2)$ \\
\hline $\mathrm{C}(14)$ & $47(2)$ & $50(2)$ & $38(2)$ & $3(2)$ & $9(2)$ & $-8(2)$ \\
\hline$C(15)$ & $53(2)$ & $79(3)$ & $51(2)$ & $-14(2)$ & $7(2)$ & $-14(3)$ \\
\hline$C(16)$ & $47(2)$ & $94(4)$ & $59(2)$ & $-10(2)$ & $11(2)$ & $-6(2)$ \\
\hline$C(17)$ & $54(2)$ & $100(4)$ & $57(2)$ & $-5(2)$ & $22(2)$ & $-7(3)$ \\
\hline $\mathrm{C}(18)$ & $59(2)$ & $88(3)$ & $53(2)$ & $-12(2)$ & $16(2)$ & $-13(3)$ \\
\hline$C(19)$ & $57(2)$ & $58(3)$ & $61(2)$ & $-7(2)$ & $17(2)$ & $-3(2)$ \\
\hline$C(20)$ & $98(3)$ & $110(5)$ & $74(3)$ & $-19(3)$ & $38(3)$ & $0(3)$ \\
\hline $\mathrm{C}(21)$ & $87(3)$ & $65(3)$ & $120(4)$ & $5(3)$ & $36(3)$ & $7(3)$ \\
\hline$C(22)$ & $77(3)$ & $178(6)$ & $126(4)$ & $-59(5)$ & $58(3)$ & $-24(4)$ \\
\hline $\mathrm{N}$ & $42(2)$ & $46(2)$ & $43(2)$ & $-3(2)$ & $8(1)$ & $9(2)$ \\
\hline $\mathrm{O}(1)$ & $40(1)$ & $63(2)$ & $56(2)$ & $-14(1)$ & $14(1)$ & $1(1)$ \\
\hline $\mathrm{O}(2)$ & $43(2)$ & $68(2)$ & $75(2)$ & $-5(2)$ & $0(1)$ & $14(2)$ \\
\hline $\mathrm{O}(3)$ & $63(2)$ & $70(2)$ & $92(2)$ & $36(2)$ & $-4(2)$ & $-12(2)$ \\
\hline $\mathrm{O}(4)$ & $46(1)$ & $60(2)$ & $48(1)$ & $12(1)$ & $2(1)$ & $-14(1)$ \\
\hline
\end{tabular}

The anisotropic displacement factor exponent takes the form:

$-2{ }^{2}\left[h^{2} \mathrm{a}^{* 2} \mathrm{U}_{11}+k^{2} \mathrm{~b} *^{2} \mathrm{U}_{22}+l^{2} \mathrm{c}^{* 2} \mathrm{U}_{33}+2 h k \mathrm{a} * \mathrm{~b} * \mathrm{U}_{12}+2 k l \mathrm{~b} * \mathrm{c} * \mathrm{U}_{23}+2 h l \mathrm{a} * \mathrm{c} * \mathrm{U}_{13}\right]$ 
Table 5. Selected Bond Lengths $(\AA)$ for $\mathbf{6 a}$ and $\mathbf{6 b}$.

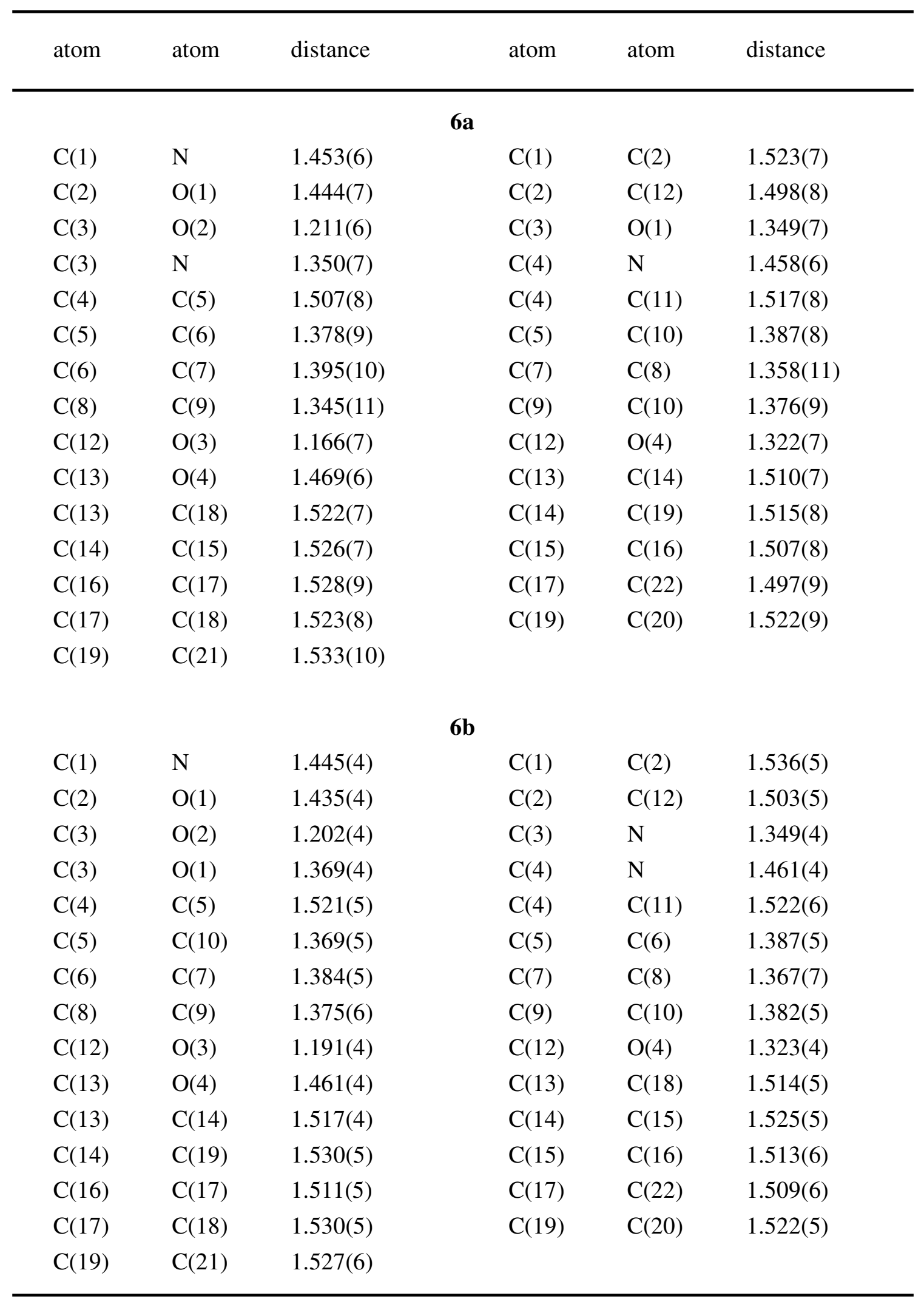


Table 6. Selected Bond Angles $\left({ }^{\circ}\right)$ for $\mathbf{6 a}$ and $\mathbf{6 b}$.

\begin{tabular}{|c|c|c|c|c|c|c|c|}
\hline atom & atom & atom & angle & atom & atom & atom & angle \\
\hline \multicolumn{8}{|c|}{$6 \mathbf{a}$} \\
\hline $\mathrm{N}$ & $\mathrm{C}(1)$ & $\mathrm{C}(2)$ & $99.7(4)$ & $\mathrm{O}(1)$ & $\mathrm{C}(2)$ & $C(12)$ & $106.7(5)$ \\
\hline $\mathrm{O}(1)$ & $\mathrm{C}(2)$ & $\mathrm{C}(1)$ & $104.0(5)$ & $\mathrm{C}(12)$ & $\mathrm{C}(2)$ & $\mathrm{C}(1)$ & $116.7(5)$ \\
\hline $\mathrm{O}(2)$ & $\mathrm{C}(3)$ & $\mathrm{O}(1)$ & $122.9(6)$ & $\mathrm{O}(2)$ & $\mathrm{C}(3)$ & $\mathrm{N}$ & $127.0(6)$ \\
\hline $\mathrm{O}(1)$ & $\mathrm{C}(3)$ & $\mathrm{N}$ & $110.1(5)$ & $\mathrm{N}$ & $\mathrm{C}(4)$ & $\mathrm{C}(5)$ & $111.4(5)$ \\
\hline $\mathrm{N}$ & $\mathrm{C}(4)$ & $\mathrm{C}(11)$ & $110.1(5)$ & $C(5)$ & $\mathrm{C}(4)$ & $\mathrm{C}(11)$ & $114.9(6)$ \\
\hline$C(6)$ & $C(5)$ & $\mathrm{C}(10)$ & $116.9(7)$ & $C(6)$ & $\mathrm{C}(5)$ & $\mathrm{C}(4)$ & $123.5(7)$ \\
\hline$C(10)$ & $C(5)$ & $\mathrm{C}(4)$ & $119.6(7)$ & $\mathrm{C}(5)$ & $\mathrm{C}(6)$ & $C(7)$ & $120.8(9)$ \\
\hline$C(8)$ & $C(7)$ & $C(6)$ & $120.3(9)$ & $\mathrm{C}(9)$ & $\mathrm{C}(8)$ & $C(7)$ & $120.0(9)$ \\
\hline$C(8)$ & $\mathrm{C}(9)$ & $C(10)$ & $120.4(9)$ & $\mathrm{C}(9)$ & $\mathrm{C}(10)$ & $\mathrm{C}(5)$ & $121.7(8)$ \\
\hline $\mathrm{O}(3)$ & $\mathrm{C}(12)$ & $\mathrm{O}(4)$ & $123.2(6)$ & $\mathrm{O}(3)$ & $\mathrm{C}(12)$ & $\mathrm{C}(2)$ & $123.6(6)$ \\
\hline $\mathrm{O}(4)$ & $\mathrm{C}(12)$ & $C(2)$ & $113.2(6)$ & $\mathrm{O}(4)$ & $\mathrm{C}(13)$ & $C(14)$ & $108.2(4)$ \\
\hline $\mathrm{O}(4)$ & $\mathrm{C}(13)$ & $\mathrm{C}(18)$ & $107.9(4)$ & $\mathrm{C}(14)$ & $C(13)$ & $\mathrm{C}(18)$ & $112.9(5)$ \\
\hline$C(13)$ & $\mathrm{C}(14)$ & $C(19)$ & $112.7(5)$ & $\mathrm{C}(13)$ & $C(14)$ & $C(15)$ & $108.4(5)$ \\
\hline$C(19)$ & $C(14)$ & $C(15)$ & $114.9(5)$ & $C(16)$ & $C(15)$ & $C(14)$ & $112.3(5)$ \\
\hline$C(15)$ & $C(16)$ & $C(17)$ & $113.3(6)$ & $\mathrm{C}(22)$ & $C(17)$ & $\mathrm{C}(18)$ & $111.7(6)$ \\
\hline$C(22)$ & $\mathrm{C}(17)$ & $C(16)$ & $112.6(7)$ & $\mathrm{C}(18)$ & $C(17)$ & $C(16)$ & $109.2(5)$ \\
\hline$C(13)$ & $\mathrm{C}(18)$ & $C(17)$ & $112.7(5)$ & $\mathrm{C}(14)$ & $C(19)$ & $\mathrm{C}(20)$ & $110.9(6)$ \\
\hline$C(14)$ & $C(19)$ & $C(21)$ & $115.2(7)$ & $\mathrm{C}(20)$ & $C(19)$ & $\mathrm{C}(21)$ & $109.7(6)$ \\
\hline$C(3)$ & $\mathrm{N}$ & $\mathrm{C}(1)$ & $110.7(5)$ & $\mathrm{C}(3)$ & $\mathrm{N}$ & $\mathrm{C}(4)$ & $121.5(5)$ \\
\hline $\mathrm{C}(1)$ & $\mathrm{N}$ & $\mathrm{C}(4)$ & $123.3(5)$ & $\mathrm{C}(3)$ & $\mathrm{O}(1)$ & $\mathrm{C}(2)$ & $108.2(5)$ \\
\hline$C(12)$ & $\mathrm{O}(4)$ & $\mathrm{C}(13)$ & $119.9(5)$ & & & & \\
\hline \multicolumn{8}{|c|}{$6 b$} \\
\hline $\mathrm{N}$ & $\mathrm{C}(1)$ & $\mathrm{C}(2)$ & $101.5(3)$ & $\mathrm{O}(1)$ & $\mathrm{C}(2)$ & $\mathrm{C}(12)$ & $109.5(3)$ \\
\hline $\mathrm{O}(1)$ & $\mathrm{C}(2)$ & $\mathrm{C}(1)$ & $105.5(2)$ & $C(12)$ & $C(2)$ & $\mathrm{C}(1)$ & $110.9(3)$ \\
\hline $\mathrm{O}(2)$ & $C(3)$ & $\mathrm{N}$ & $128.6(3)$ & $\mathrm{O}(2)$ & $\mathrm{C}(3)$ & $\mathrm{O}(1)$ & $122.0(4)$ \\
\hline $\mathrm{N}$ & $\mathrm{C}(3)$ & $\mathrm{O}(1)$ & $109.4(3)$ & $\mathrm{N}$ & $\mathrm{C}(4)$ & $C(5)$ & $112.4(3)$ \\
\hline $\mathrm{N}$ & $\mathrm{C}(4)$ & $\mathrm{C}(11)$ & 109.7(3) & $\mathrm{C}(5)$ & $C(4)$ & $\mathrm{C}(11)$ & $113.2(4)$ \\
\hline$C(10)$ & $\mathrm{C}(5)$ & $\mathrm{C}(6)$ & $117.5(4)$ & $\mathrm{C}(10)$ & $C(5)$ & $\mathrm{C}(4)$ & $122.7(3)$ \\
\hline$C(6)$ & $C(5)$ & $\mathrm{C}(4)$ & $119.7(4)$ & $C(7)$ & $\mathrm{C}(6)$ & $C(5)$ & $121.5(4)$ \\
\hline $\mathrm{C}(8)$ & $C(7)$ & $C(6)$ & $119.6(5)$ & $C(7)$ & $\mathrm{C}(8)$ & $\mathrm{C}(9)$ & $120.1(4)$ \\
\hline$C(8)$ & $\mathrm{C}(9)$ & $\mathrm{C}(10)$ & $119.6(5)$ & $C(5)$ & $C(10)$ & $\mathrm{C}(9)$ & $121.8(4)$ \\
\hline
\end{tabular}


Table 6. Selected Bond Angles $\left(^{\circ}\right)$ for $\mathbf{6 a}$ and $\mathbf{6 b}$. (continued)

\begin{tabular}{llllllll}
\hline atom & atom & atom & angle & atom & atom & atom & angle \\
\hline & & & & & & & \\
$\mathrm{O}(3)$ & $\mathrm{C}(12)$ & $\mathrm{O}(4)$ & $125.2(4)$ & $\mathrm{O}(3)$ & $\mathrm{C}(12)$ & $\mathrm{C}(2)$ & $125.1(3)$ \\
$\mathrm{O}(4)$ & $\mathrm{C}(12)$ & $\mathrm{C}(2)$ & $109.5(3)$ & $\mathrm{O}(4)$ & $\mathrm{C}(13)$ & $\mathrm{C}(18)$ & $106.8(3)$ \\
$\mathrm{O}(4)$ & $\mathrm{C}(13)$ & $\mathrm{C}(14)$ & $108.0(3)$ & $\mathrm{C}(18)$ & $\mathrm{C}(13)$ & $\mathrm{C}(14)$ & $113.3(3)$ \\
$\mathrm{C}(13)$ & $\mathrm{C}(14)$ & $\mathrm{C}(15)$ & $108.8(3)$ & $\mathrm{C}(13)$ & $\mathrm{C}(14)$ & $\mathrm{C}(19)$ & $112.9(3)$ \\
$\mathrm{C}(15)$ & $\mathrm{C}(14)$ & $\mathrm{C}(19)$ & $114.4(3)$ & $\mathrm{C}(16)$ & $\mathrm{C}(15)$ & $\mathrm{C}(14)$ & $113.0(4)$ \\
$\mathrm{C}(17)$ & $\mathrm{C}(16)$ & $\mathrm{C}(15)$ & $111.7(3)$ & $\mathrm{C}(22)$ & $\mathrm{C}(17)$ & $\mathrm{C}(16)$ & $112.4(4)$ \\
$\mathrm{C}(22)$ & $\mathrm{C}(17)$ & $\mathrm{C}(18)$ & $111.4(4)$ & $\mathrm{C}(16)$ & $\mathrm{C}(17)$ & $\mathrm{C}(18)$ & $108.7(3)$ \\
$\mathrm{C}(13)$ & $\mathrm{C}(18)$ & $\mathrm{C}(17)$ & $112.5(4)$ & $\mathrm{C}(20)$ & $\mathrm{C}(19)$ & $\mathrm{C}(21)$ & $110.0(4)$ \\
$\mathrm{C}(20)$ & $\mathrm{C}(19)$ & $\mathrm{C}(14)$ & $111.7(3)$ & $\mathrm{C}(21)$ & $\mathrm{C}(19)$ & $\mathrm{C}(14)$ & $114.5(4)$ \\
$\mathrm{C}(3)$ & $\mathrm{N}$ & $\mathrm{C}(1)$ & $112.8(3)$ & $\mathrm{C}(3)$ & $\mathrm{N}$ & $\mathrm{C}(4)$ & $122.2(3)$ \\
$\mathrm{C}(1)$ & $\mathrm{N}$ & $\mathrm{C}(4)$ & $124.0(3)$ & $\mathrm{C}(3)$ & $\mathrm{O}(1)$ & $\mathrm{C}(2)$ & $110.0(3)$ \\
$\mathrm{C}(12)$ & $\mathrm{O}(4)$ & $\mathrm{C}(13)$ & $119.0(3)$ & & & & \\
& & & & & & & \\
\hline
\end{tabular}

